\title{
The ESO Slice Project (ESP) galaxy redshift survey ${ }^{\star}$
}

\section{The sample}

\author{
G. Vettolani ${ }^{1}$, E. Zucca ${ }^{2,1}$, R. Merighi ${ }^{2}$, M. Mignoli ${ }^{2}$, D. Proust ${ }^{3}$, G. Zamorani ${ }^{2,1}$, A. Cappi $^{2}$, L. Guzzo $^{4}$, \\ D. Maccagni $^{5}$, M. Ramella ${ }^{6}$, G.M. Stirpe ${ }^{2}$, A. Blanchard ${ }^{7}$, V. Cayatte ${ }^{3}$, C. Collins ${ }^{8}$, H. MacGillivray ${ }^{9}$, \\ S. Maurogordato ${ }^{10,3}$, R. Scaramella ${ }^{11}$, C. Balkowski ${ }^{3}$, G. Chincarini ${ }^{5,12}$, and P. Felenbok ${ }^{3}$ \\ 1 Istituto di Radioastronomia del CNR, via Gobetti 101, 40129 Bologna, Italy \\ 2 Osservatorio Astronomico di Bologna, via Zamboni 33, 40126 Bologna, Italy \\ 3 Observatoire de Paris, DAEC, Unité associée au CNRS, D0173 et à l'Université Paris 7, 5 Place J. Janssen, 92195 Meudon, \\ France \\ 4 Osservatorio Astronomico di Brera, via Bianchi 46, 22055 Merate (LC), Italy \\ 5 Istituto di Fisica Cosmica e Tecnologie Relative, via Bassini 15, 20133 Milano, Italy \\ 6 Osservatorio Astronomico di Trieste, via Tiepolo 11, 34131 Trieste, Italy \\ 7 Université L. Pasteur, Observatoire Astronomique, 11 rue de l'Université, 67000 Strasbourg, France \\ 8 Astrophysics Research Institute, Liverpool John-Moores University, Byrom Street, Liverpool L3 3AF, UK \\ 9 Royal Observatory Edinburgh, Blackford Hill, Edinburgh EH9 3HJ, UK \\ 10 CERGA, Observatoire de la Côte d'Azur, BP. 229, 06304 Nice Cedex 4, France \\ 11 Osservatorio Astronomico di Roma, via Osservatorio 2, 00040 Monteporzio Catone (RM), Italy \\ 12 Università degli Studi di Milano, via Celoria 16, 20133 Milano, Italy
}

Received November 12; accepted December 22, 1997

\begin{abstract}
The ESO Slice Project (ESP) is a galaxy redshift survey extending over about 23 square degrees, in a region near the South Galactic Pole. The survey is $\sim 85 \%$ complete to the limiting magnitude $b_{\mathrm{J}}=19.4$ and consists of 3342 galaxies with redshift determination.

The ESP survey is intermediate between shallow, wide angle samples and very deep, one--dimensional pencil beams; the spanned volume is $\sim 510^{4} h^{-3} \mathrm{Mpc}^{3}$ at the sensitivity peak $(z \sim 0.1)$.

In this paper we present the description of the observations and of the data reduction, the ESP redshift catalogue and the analysis of the quality of the velocity determinations.
\end{abstract}

\footnotetext{
Send offprint requests to: Elena Zucca

(zucca@astbo1.bo.cnr.it)

* based on observations collected at the European Southern Observatory, La Silla, Chile. Table 3 is only available (and Table 2 is also available) in electronic form at the CDS via anonymous ftp to cdsarc.u-strasbg.fr (130.79.128.5) or via http://cdsweb.u-strasbg.fr/Abstract.html
}

Key words: galaxies: distances and redshifts - cosmology: observations; large-scale structure of the Universe

\section{Introduction}

The ESO Slice Project (ESP) galaxy redshift survey, which is described in Vettolani et al. (1997, hereafter Paper I), extends over a strip of $\alpha \times \delta=22^{\circ} \times 1^{\circ}$, plus a nearby area of $5^{\circ} \times 1^{\circ}$, five degrees west of the main strip, in the South Galactic Pole region. The right ascension limits are $22^{\mathrm{h}} 30^{\mathrm{m}}$ and $01^{\mathrm{h}} 20^{\mathrm{m}}$, at a mean declination of $-40^{\circ} 15^{\prime}(1950)$. We have covered this region with a regular grid of adjacent circular fields, with a diameter of 32 arcmin each, corresponding to the field of view of the multifiber spectrograph OPTOPUS (Lund 1986; Avila et al. 1989 ) at the $3.6 \mathrm{~m}$ ESO telescope. The total solid angle of the spectroscopic survey is 23.2 square degrees.

This paper presents the survey data (photometry, spectroscopy, completeness, etc.) which are necessary for a comprehensive study of the sample. It is organized as 
follows: in Sect. 2 we describe the photometric sample, in Sect. 3 the observations and data reduction and in Sect. 4 the redshift determination. In Sect. 5 we present the catalogue, in Sect. 6 we discuss the possible biases in the sample and the velocity errors, and finally Sect. 7 provides a summary.

\section{The photometric sample}

The galaxy catalogue has been extracted from the Edinburgh-Durham Southern Galaxy catalogue (hereafter EDSGC, Heydon-Dumbleton et al. 1988, 1989) which has been obtained from COSMOS (MacGillivray \& Stobie 1984) scans of SERC J survey plates. The EDSGC has a $95 \%$ completeness at $b_{\mathrm{J}} \leq 20.0$ and an estimated stellar contamination $\leq 10 \%$ (Heydon-Dumbleton et al. 1989). The systematic intra-plate photometric errors are less than $\sim 0.03$ magnitudes and the inter-plate limiting magnitude variation is less than $\sim 0.04$ magnitudes (HeydonDumbleton et al. 1988).

In order to obtain an external estimate of the photometric accuracy of the sample, we have compared the EDSGC magnitudes with CCD photometry, from a multicolour survey of a subsample of ESP galaxies (Garilli et al. in preparation). Preliminary analysis of these data, obtained with the $0.9 \mathrm{~m}$ Dutch/ESO telescope for about 80 galaxies in the magnitude range $16.5 \leq b_{\mathrm{J}} \leq 19.4$, shows a linear relation between $b_{\mathrm{J}}(\mathrm{EDSGC})$ and $m_{\mathrm{B}}(\mathrm{CCD})$, with a dispersion $\left(\sigma_{\mathrm{M}}\right)$ of about 0.2 magnitudes around the fit. Since the CCD pointings cover the entire right ascension range of our survey, this $\sigma_{\mathrm{M}}$ includes both statistical errors within single plates and possible plate-to-plate zero point variations.

All EDSGC galaxies in the ESP region have been examined visually on high contrast enlarged $(\times 8.4)$ reproductions of the ESO-SERC atlas plates. A small percentage $(\lesssim 2 \%)$ of the catalogue entries was discarded on the basis of the visual inspection (portions of star spikes, bright galaxy spiral arms "broken" into multiple entries, etc...). These objects, although listed in the original EDSGC catalogue, were not included in the ESP sample and therefore are not listed in the final catalogue.

At visual examination 287 objects appeared as formed by two components, not separated by the EDSGC deblending algorithm. These 287 objects, which on average have a magnitude distribution fainter than that of the total sample, could be either two galaxies almost in contact, or a galaxy with a nearby star, or a pair of stars, or a galaxy with a prominent HII region. Being difficult to determine the nature of each object on the basis of the visual inspection and to estimate the magnitude of each component separately, we have kept these objects as single entries in the ESP catalogue in order not to bias a priori the sample.

The number of objects in the photometric ESP sample is 4487 .
Table 1. OPTOPUS-MEFOS observing log

\begin{tabular}{cclc}
\hline Run & Date & Set-up & \# Obs. Fields \\
\hline$\# 1$ & $02-05 / 09 / 91$ & OPTOPUS+TEK\#16 & 18 \\
$\# 2$ & $12-15 / 10 / 91$ & OPTOPUS+TEK\#16 & 16 \\
$\# 3$ & $21-24 / 09 / 92$ & OPTOPUS+TEK\#16 & 22 \\
$\# 4$ & $24-27 / 10 / 92$ & OPTOPUS+TEK\#16 & 19 \\
$\# 5$ & $08-12 / 09 / 93$ & OPTOPUS+TEK\#32 & 30 \\
$\# 6$ & $16-18 / 10 / 93$ & OPTOPUS+TEK\#32 & 15 \\
$\# 7$ & $27-30 / 10 / 94$ & MEFOS+TEK\#32 & 15 \\
\hline
\end{tabular}

\section{Observations and data reduction}

\subsection{Instrumentation}

Spectroscopic observations were performed at the ESO $3.6 \mathrm{~m}$ telescope in La Silla. The main body of data was obtained in six observing runs with the OPTOPUS multifiber spectrograph at the Cassegrain focus (Lund 1986; Avila et al. 1989). Further observations were obtained in 1994 with the multifiber spectrograph MEFOS (Avila et al. 1995; Felenbok et al. 1997) at the prime focus of the same telescope. A log of the observations is given in Table 1.

OPTOPUS has a bundle of 50 optical fibers (Polymicro FHP with $320 \mu \mathrm{m}$ core and enhanced blue sensitivity), which are manually plugged into aluminium plates with holes and fiberholders at the galaxy positions. These positions are corrected for differential refraction between the wavelength centre of the autoguider sensor and the centre of the spectral range, at the expected zenith angle of observation. The plates are mounted at the Cassegrain focal plane and have a diameter of 32 arcmin on the sky. The core of the fibers corresponds to a sky aperture of 2.3 arcsec in diameter. At the opposite end the fibers are coupled to an $f$ / 8 collimator of the Boller \& Chivens spectrograph, forming the entrance slit.

Once mounted, the plates are centred using four relatively bright stars at the periphery of the plate, observed through fibers going to an intensified camera. Exposure guiding is accomplished by means of two fiber bundles, which are used to image two relatively bright stars in the field through holes in the plate. The mechanical constraints are such that the minimum object-to-object separation is 24.6 arcsec and minimum object-to-guide star separation is 64.3 arcsec.

For the present program we used the ESO grating \# 15 (300 lines $\mathrm{mm}^{-1}$ and blaze angle of $4^{\circ} 18^{\prime}$ ), allowing a dispersion of $174 \AA \mathrm{mm}^{-1}$ with an $f / 1.9$ blue camera in the wavelength range $3750-6150 \AA$. Initially the detector was a Tektronix $512 \times 512 \mathrm{CCD}$ with a pixel size of 27 microns, corresponding to $4.5 \AA$ / pixel, a velocity bin of $\simeq 270 \mathrm{~km} / \mathrm{s}$ at $5000 \AA$. After the October 1992 observing run, the detector was replaced with a new Tektronix 
thinned, back-illuminated CCD (ESO \#32), which also had a pixel size of 27 microns, giving a similar spectral coverage but a better blue sensitivity.

The observing time for each field was one hour, split into two 30 mins exposures to ease the removal of cosmic rays. Keeping the telescope in the same position, exposures with a quartz-halogen lamp as well as of a Helium comparison lamp were taken immediately before and after the science exposures. Depending on the length of the night we observed from 4 to 6 different fields per night choosing the sequence of the fields to be observed in such a way so as to minimize the zenith distance and hence the differential refraction.

Further observations were accomplished in October 1994 with the multifiber spectrograph MEFOS, at the prime focus of the $3.6 \mathrm{~m}$ telescope.

The MEFOS Spectrograph has 30 robotic arms in a "fishermen-around-the-pond" configuration over a field of one degree diameter. One arm is used for guiding purposes, while the other 29 arms carry two spectroscopic fibers each, one fiber for the object and one for the sky. Because of the prime focus scale, the optical fibers (Polymicro FBP) have a $135 \mu \mathrm{m}$ core, corresponding to a sky aperture of 2.6 arcsec. The object-sky fiber separation is 60 arcsec, and the minimum object-to-object distance is 28 arcsec. Each arm also carries an imaging fiber bundle which is used to accurately centre the fibers on the targets. The output ends of the spectroscopic fibers are arranged in a line so as to form the entrance slit of the same Boller \& Chivens spectrograph used for OPTOPUS. With a new optimized $f / 3.03$ collimator the spectrographCCD configuration was the same as used for the previous OPTOPUS observations.

\subsection{Data reduction}

The data reduction was performed using the APEXTRACT package as implemented in $\operatorname{IRAF}^{1}$. For each exposure first we identified and followed the spectra on the white lamp frame. Then the solutions obtained for the white lamps were applied to extract the one-dimensional spectra both in the calibration and science frames. Using APEXTRACT we performed an "optimal extraction" (Horne 1986), also detecting and replacing the highly deviant bad pixels and cosmic ray hits.

The individual wavelength calibration for each fiber is derived from the corresponding arc spectrum. We used a fourth-order polynomial fit with typical rms errors of $0.2-$ $0.3 \AA$. The accuracy of the calibration was estimated from the measured wavelengths of two [OI] sky lines $(\lambda \lambda 5577$, 6300 ), which were always within $\pm 1 \AA$ (less than a quarter of pixel) from their expected positions.

\footnotetext{
${ }^{1}$ IRAF is distributed by the National Optical Astronomy Observatories, which is operated by AURA Inc. for the NSF.
}

A critical point in fiber spectroscopy is the sky subtraction because it is impossible to measure the background locally and through the same aperture (fiber) of the object (see Parry \& Carrasco 1990 or Wyse \& Gilmore 1992 for a detailed discussion). We adopted the following strategy. For OPTOPUS observations we dedicated at least four apertures to the sky in each field. We defined predetermined positions on each plate which were verified not to have any object at the limit of SERC J plates. For MEFOS the sky fibers were as many as the target objects. With both instruments we performed a "mean-sky" subtraction method (Cuby \& Mignoli 1994).

The main problem is the fiber throughput determination, because of the different transmission of each waveguide which could also vary from one exposure to the other. The most intense and isolated sky line in our spectral range is the [OI]5577 emission line, and we used its flux as an estimator of the fiber trasmittance. We directly measured the line counts on the calibrated spectra by mean of a Gaussian profile fit, after subtracting the underlying continuum (due to both sky and object, if any, contribution). Under the assumption that the intensity of the night spectrum does not vary appreciably within the telescope field, the flux of this sky line is the best estimator of the fiber throughput. In fact it is easily measurable both in the sky and object spectra and it is subject to the same temporal transmittance variations as the object flux (e.g. due to the differential fiber flexures during the exposure). In order to reveal possible contaminations (cosmic ray hits or object emission/absorption lines) that could affect the fiber throughput estimate, we also computed the mean and the dispersion of the ratio between the peak of the continuum-subtracted counts of the [OI]5577 and the same quantity for the [OI]6300 sky line: spectra with unusual value of this ratio were re-examined to check the reason for the discrepancy. In all the relatively few cases in which this has occurred, the problem was overcome by interactively fitting the [OI]5577 line and eliminating the cosmic ray contaminations, or was due to an underestimate of the sky $[\mathrm{OI}] 6300$ emission because of the coincident absorption feature in stellar objects.

After the normalization to account for the relative fiber transmission, a "mean sky" was determined using all the sky spectra (four or more for OPTOPUS, up to 29 for MEFOS) and subtracted from all the "object plus sky" spectra of the same exposure. This procedure was repeated separately for each of the two 30 mins exposures. The comparison of the two exposures of the same field revealed the remaining cosmic rays spikes, which were eliminated by interpolating the adjacent pixels.

The sky-subtraction accuracy is difficult to gauge. The residual counts in the sky-subtracted sky spectra provide a good estimator of this, but caution is necessary: indeed, the simple rms value of the sky-subtracted sky spectrum could overestimate the quality of the sky subtraction because it does not take account for "bias residual" if the 
average background has been over/underestimated (see Wyse \& Gilmore 1992). Therefore we adopted, as estimator of the sky-subtraction accuracy, the "average absolute sky-residuals" quantity

$Q_{i}=\left\langle\left|\frac{\mathrm{sky}_{i}-\left\langle\mathrm{sky}_{i}\right.}{\left\langle\mathrm{sky}_{i}\right.}\right|\right\rangle_{\lambda}$

This quantity has been measured, resulting in the range of $2.8 \div 7.5 \%$ with a typical value of $4 \%$.

\section{Redshift determination}

Redshifts were measured for absorption-featured spectra using the task XCSAO (Kurtz et al. 1992), a crosscorrelation program (Tonry \& Davis 1979) developed at the Smithsonian Astrophysical Observatory as a part of the RVSAO package, a contributed IRAF package.

The program allows the user to adapt the several working parameters to the characteristics of its own data-set. It is in fact possible to control if and how to rebin the data in log wavelength, to set an initial guess for the radial velocity, to define the dimension of the apodization region to be applied to the spectra, to fit and subtract the continuum from both spectra and templates, to choose the appropriate Fourier filtering and how to fit the peak of the correlation function. Once these parameters are defined, the program can be run in batch mode. An interactive graphical mode is available, during which all the main parameters can be modified and tested.

Redshifts for emission line objects were instead determined using EMSAO (Mink \& Wyatt 1995), the XCSAO companion task. EMSAO was developed to find emission lines automatically, to compute redshifts for each identified line and to combine them into a single radial velocity. The results may be graphically displayed or printed. The graphic cursor can be used to interactively change fit and display parameters.

\subsection{Templates}

A crucial point for minimizing the error of the crosscorrelation is the construction of a good template system, i.e. a set of spectra closely reproducing the characteristics of the observed spectra, having for example a range of relative absorption line depths similar to that of the sample galaxies of different morphological types.

After the first observing run we realized that some of the spectroscopic stars, which were misclassified as galaxies in the EDSGC, could represent such a set of templates.

A system of eight stars (6 of $\mathrm{F}$ type and 2 of $\mathrm{K}$ type) was chosen and used. Note that the use of such cold stars is a valuable criterion for cross-correlation procedure, since the profile of strong lines such as the calcium $K$ and $H$, the $G$ band, $\mathrm{H} \beta$ and $\mathrm{MgI}$ is not affected by gravity and/or metal abundance: it remains narrow, with a Doppler core and Stark broadened wings.

We made the assumption that such a group of stars could define a self consistent system with an average radial velocity of zero $\mathrm{km} / \mathrm{s}$. This assumption has been verified cross-correlating against this template system three standard radial velocity stars we purposely observed with OPTOPUS. We measured a zero point shift of $-7.4 \pm$ $3.8 \mathrm{~km} / \mathrm{s}$; this correction has not been applied to the data of the catalogue.

\subsection{Redshift measurement}

Absorption spectra were measured with XCSAO and we decided to adopt as the "absorption velocity" the one associated with the minimum error in $\mathrm{km} / \mathrm{s}$ from the crosscorrelation against the eight stellar templates. In the great majority of cases, this coincided also with the maximum $R$ parameter of Tonry \& Davis (1979). Generally, the best performing templates were the $\mathrm{F}$ stars, but in a few cases better measurements were obtained from the two $\mathrm{K}$ templates.

Spectra showing both absorption and emission features were generally measured with both tasks (XCSAO and EM$\mathrm{SAO}$ ). Emission features were manually excised prior to performing the cross-correlation analysis.

As a consistency check, all spectra were examined also visually, in order to verify the assigned velocity. A few examples of spectra of different quality are shown in Fig. 1.

\section{Catalogue presentation}

In Table 2 we list the fields we observed with OPTOPUS (see Sect. 1 and Paper I) and their properties. Column (1) gives the number of the field, whose centre coordinates $(\alpha$ and $\delta$ ) are reported in Cols. (2) and (3). Col. (4) gives the total number of objects classified as galaxies in the photometric catalogue $\left(N_{\mathrm{T}}\right)$, while Cols. $(5),(6),(7)$ and (8) give the number of redshifts $\left(N_{\mathrm{Z}}\right)$, of not-observed objects $\left(N_{\mathrm{NO}}\right)$, of failed spectra (i.e. not useful to obtain a redshift determination, $\left.N_{\mathrm{F}}\right)$ and of stars $\left(N_{\mathrm{S}}\right)$, respectively. From these numbers the redshift completeness of each field can be derived (see also Paper I and Zucca et al. 1997) as

$\frac{N_{\mathrm{Z}}}{N_{\mathrm{T}}-N_{\mathrm{S}}-0.122 * N_{\mathrm{NO}}}$.

This equation assumes that the failed spectra correspond to galaxies, because stars, being point-like objects, have on average a better signal-to-noise ratio than galaxies, and that the percentage of stars in not-observed objects spectra is the same as in the spectroscopic sample (i.e. $\sim 12.2 \%$ ).

Note that the field centres are separated by 30 arcmin both in right ascension and declination whilst the OPTOPUS fields have a diameter of 32 arcmin. Therefore 
Table 2. OPTOPUS fields (available also in electronic form)

\begin{tabular}{|c|c|c|c|c|c|c|c|c|c|c|c|c|c|c|c|}
\hline$\#$ & $\begin{array}{r}\alpha \\
(1950)\end{array}$ & $\begin{array}{r}\delta \\
(1950)\end{array}$ & $N_{\mathrm{T}}$ & $N_{\mathrm{Z}}$ & $N_{\mathrm{NO}}$ & $N_{\mathrm{F}}$ & $N_{\mathrm{S}}$ & $\#$ & $\begin{array}{r}\alpha \\
(1950)\end{array}$ & $\begin{array}{r}\delta \\
(1950)\end{array}$ & $N_{\mathrm{T}}$ & $N_{\mathrm{Z}}$ & $N_{\mathrm{NO}}$ & $N_{\mathrm{F}}$ & $N_{\mathrm{S}}$ \\
\hline 1 & 223118.3 & -400000 & 70 & 36 & 25 & 2 & 7 & 101 & 223118.3 & -403000 & 74 & 36 & 28 & 5 & 5 \\
\hline 2 & 223355.0 & -400000 & 56 & 33 & 11 & 8 & 4 & 102 & 223355.0 & -403000 & 62 & 39 & 19 & 1 & 3 \\
\hline 3 & 223631.6 & -400000 & 72 & 33 & 30 & 5 & 4 & 103 & 223631.6 & -403000 & 42 & 34 & 3 & 1 & 4 \\
\hline 4 & 223908.3 & -400000 & 77 & 28 & 34 & 9 & 6 & 104 & 223908.3 & -403000 & 36 & 30 & 2 & 1 & 3 \\
\hline 5 & 224144.9 & -400000 & 80 & 41 & 32 & 1 & 6 & 105 & 224144.9 & -403000 & 57 & 34 & 12 & 4 & 7 \\
\hline 6 & 224421.6 & -400000 & 53 & 32 & 10 & 1 & 10 & 106 & 224421.6 & -403000 & 53 & 23 & 16 & 8 & 6 \\
\hline 7 & 224658.2 & -400000 & 69 & 34 & 25 & 1 & 9 & 107 & 224658.2 & -403000 & 92 & 37 & 43 & 0 & 12 \\
\hline 8 & 224934.9 & -400000 & 49 & 37 & 4 & 3 & 5 & 108 & 224934.9 & -403000 & 54 & 31 & 9 & 2 & 12 \\
\hline 9 & 225211.5 & -400000 & 43 & 25 & 1 & 3 & 14 & 109 & 225211.5 & -403000 & 57 & 30 & 13 & 6 & 8 \\
\hline 21 & 232331.3 & -400000 & 27 & 19 & 0 & 0 & 8 & 121 & 232331.3 & -403000 & 55 & 37 & 5 & 2 & 11 \\
\hline 22 & 232608.0 & -400000 & 43 & 33 & 1 & 0 & 9 & 122 & 232608.0 & -403000 & 42 & 32 & 0 & 3 & 7 \\
\hline 23 & 232844.6 & -400000 & 32 & 30 & 0 & 2 & 0 & 123 & 232844.6 & -403000 & 25 & 21 & 0 & 0 & 4 \\
\hline 24 & 233121.2 & -400000 & 36 & 28 & 1 & 5 & 2 & 124 & 233121.2 & -403000 & 42 & 36 & 2 & 3 & 1 \\
\hline 25 & 233357.9 & -400000 & 48 & 37 & 1 & 2 & 8 & 125 & 233357.9 & -403000 & 39 & 34 & 0 & 0 & 5 \\
\hline 26 & 233634.5 & -400000 & 36 & 26 & 2 & 4 & 4 & 126 & 233634.5 & -403000 & 31 & 24 & 1 & 1 & 5 \\
\hline 27 & 233911.2 & -400000 & 24 & 9 & 1 & 6 & 8 & 127 & 233911.2 & -403000 & 35 & 28 & 1 & 0 & 6 \\
\hline 28 & 234147.8 & -400000 & 22 & 17 & 1 & 2 & 2 & 128 & 234147.8 & -403000 & 29 & 21 & 0 & 0 & 8 \\
\hline 29 & 234424.5 & -400000 & 24 & 23 & 0 & 0 & 1 & 129 & 234424.5 & -403000 & 15 & 13 & 0 & 0 & 2 \\
\hline 30 & 234701.1 & -400000 & 19 & 16 & 0 & 1 & 2 & 130 & 234701.1 & -403000 & 23 & 19 & 1 & 0 & 3 \\
\hline 31 & 234937.8 & -400000 & 32 & 28 & 1 & 0 & 3 & 131 & 234937.8 & -403000 & 36 & 33 & 1 & 0 & 2 \\
\hline 32 & 235214.4 & -400000 & 48 & 43 & 0 & 0 & 5 & 132 & 235214.4 & -403000 & 22 & 19 & 0 & 0 & 3 \\
\hline 33 & 235451.1 & -400000 & 29 & 21 & 1 & 2 & 5 & 133 & 235451.1 & -403000 & 42 & 35 & 2 & & 4 \\
\hline 34 & 235727.7 & -400000 & 56 & 34 & 9 & 1 & 12 & 134 & 235727.7 & -403000 & 32 & 18 & 2 & 0 & 12 \\
\hline 35 & 000004.4 & -400000 & 38 & 33 & 0 & 1 & 4 & 135 & 000004.4 & -403000 & 34 & 27 & 0 & 0 & 7 \\
\hline 36 & 000241.0 & -400000 & 38 & 24 & 1 & 1 & 12 & 136 & 000241.0 & -403000 & 53 & 42 & 4 & 2 & 5 \\
\hline 37 & $\begin{array}{lll}00 & 05 & 17.7\end{array}$ & -400000 & 39 & 33 & 2 & 1 & 3 & 137 & $\begin{array}{lll}00 & 05 & 17.7\end{array}$ & -403000 & 3 & 25 & 2 & & 3 \\
\hline 38 & 000754.3 & -400000 & 29 & 26 & 1 & 2 & 0 & 138 & 000754.3 & -403000 & 18 & 15 & 1 & 0 & 2 \\
\hline 39 & 001031.0 & -400000 & 37 & 25 & 2 & 3 & 7 & 139 & 001031.0 & -403000 & 25 & 20 & 1 & 3 & 1 \\
\hline 40 & 001307.6 & -400000 & 53 & 46 & 1 & 4 & 2 & 140 & 001307.6 & -403000 & 46 & 35 & 6 & 4 & 1 \\
\hline 41 & 001544.3 & -400000 & 47 & 38 & 2 & 4 & 3 & 141 & 001544.3 & -403000 & 39 & 33 & 3 & 2 & 1 \\
\hline 42 & $\begin{array}{lll}00 & 18 & 20.9\end{array}$ & -400000 & 36 & 29 & 5 & 1 & 1 & 142 & $\begin{array}{llll}00 & 18 & 20.9\end{array}$ & -403000 & 33 & 30 & 0 & 0 & 3 \\
\hline 43 & 002057.6 & -400000 & 33 & 22 & 0 & 11 & 0 & 143 & 002057.6 & -403000 & 68 & 63 & 2 & 0 & 3 \\
\hline 44 & 002334.2 & -400000 & 49 & 40 & 6 & 0 & 3 & 144 & 002334.2 & -403000 & 43 & 38 & 4 & 0 & 1 \\
\hline 45 & $\begin{array}{lll}00 & 26 & 10.9\end{array}$ & -400000 & 40 & 29 & 2 & 7 & 2 & 145 & 002610.9 & -403000 & 25 & 25 & 0 & 0 & 0 \\
\hline 46 & 002847.5 & -400000 & 24 & 15 & 0 & 7 & 2 & 146 & 002847.5 & -403000 & 27 & 22 & 2 & 0 & 3 \\
\hline 47 & 003124.2 & -400000 & 23 & 18 & 1 & 2 & 2 & 147 & 003124.2 & -403000 & 25 & 20 & 1 & 1 & 3 \\
\hline 48 & 003400.8 & -400000 & 36 & 29 & 1 & 4 & 2 & 148 & 003400.8 & -403000 & 3 & 23 & 1 & 2 & 5 \\
\hline 49 & 003637.5 & -400000 & 28 & 22 & 0 & 0 & 6 & 149 & 003637.5 & -403000 & 21 & 18 & 1 & 0 & 2 \\
\hline 50 & 003914.1 & -400000 & 24 & 21 & 0 & 2 & 1 & 150 & 003914.1 & -403000 & 20 & 18 & 0 & 0 & 2 \\
\hline 51 & 004150.8 & -400000 & 54 & 44 & 2 & 2 & 6 & 151 & 004150.8 & -403000 & 30 & 28 & 0 & 0 & 2 \\
\hline 52 & 004427.4 & -400000 & 44 & 37 & 1 & 1 & 5 & 152 & 004427.4 & -403000 & 42 & 35 & 0 & 2 & 5 \\
\hline 53 & 004704.1 & -400000 & 59 & 58 & 0 & 0 & 1 & 153 & 004704.1 & -403000 & 43 & 37 & 3 & 1 & 2 \\
\hline 54 & 004940.7 & -400000 & 48 & 42 & 1 & 1 & 4 & 154 & 004940.7 & -403000 & 38 & 31 & 1 & 3 & 3 \\
\hline 55 & 005217.4 & -400000 & 61 & 54 & 1 & 4 & 2 & 155 & 005217.4 & -403000 & 37 & 24 & 2 & 1 & 10 \\
\hline 56 & 005454.0 & -400000 & 39 & 31 & 1 & 1 & 6 & 156 & 005454.0 & -403000 & 40 & 36 & 0 & 2 & 2 \\
\hline 57 & 005730.7 & -400000 & 60 & 56 & 0 & 0 & 4 & 157 & 005730.7 & -403000 & 67 & 45 & 6 & 3 & 13 \\
\hline 58 & 010007.3 & -400000 & 47 & 42 & 0 & 2 & 3 & 158 & $\begin{array}{lll}01 & 00 & 07.3\end{array}$ & -403000 & 48 & 40 & 3 & 0 & 5 \\
\hline 59 & 010244.0 & -400000 & 83 & 78 & 0 & 0 & 5 & 159 & 010244.0 & -403000 & 59 & 48 & 5 & 2 & 4 \\
\hline 60 & 010520.6 & -400000 & 40 & 33 & 2 & 0 & 5 & 160 & 010520.6 & -403000 & 74 & 65 & 4 & 2 & 3 \\
\hline 61 & 010757.3 & -400000 & 33 & 21 & 1 & 4 & 7 & 161 & 010757.3 & -403000 & 43 & 31 & 0 & 6 & 6 \\
\hline 62 & 011033.9 & -400000 & 28 & 23 & 1 & 0 & 4 & 162 & 011033.9 & -403000 & 34 & 31 & 1 & 0 & 2 \\
\hline 63 & 011310.6 & -400000 & 31 & 26 & 0 & 2 & 3 & 163 & 011310.6 & -403000 & 34 & 27 & 1 & 1 & 5 \\
\hline 64 & 011547.2 & -400000 & 26 & 19 & 1 & 2 & 4 & 164 & 011547.2 & -403000 & 36 & 29 & 1 & 1 & 5 \\
\hline 65 & 011823.9 & -400000 & 55 & 41 & 4 & 2 & 8 & & & & & & & & \\
\hline
\end{tabular}



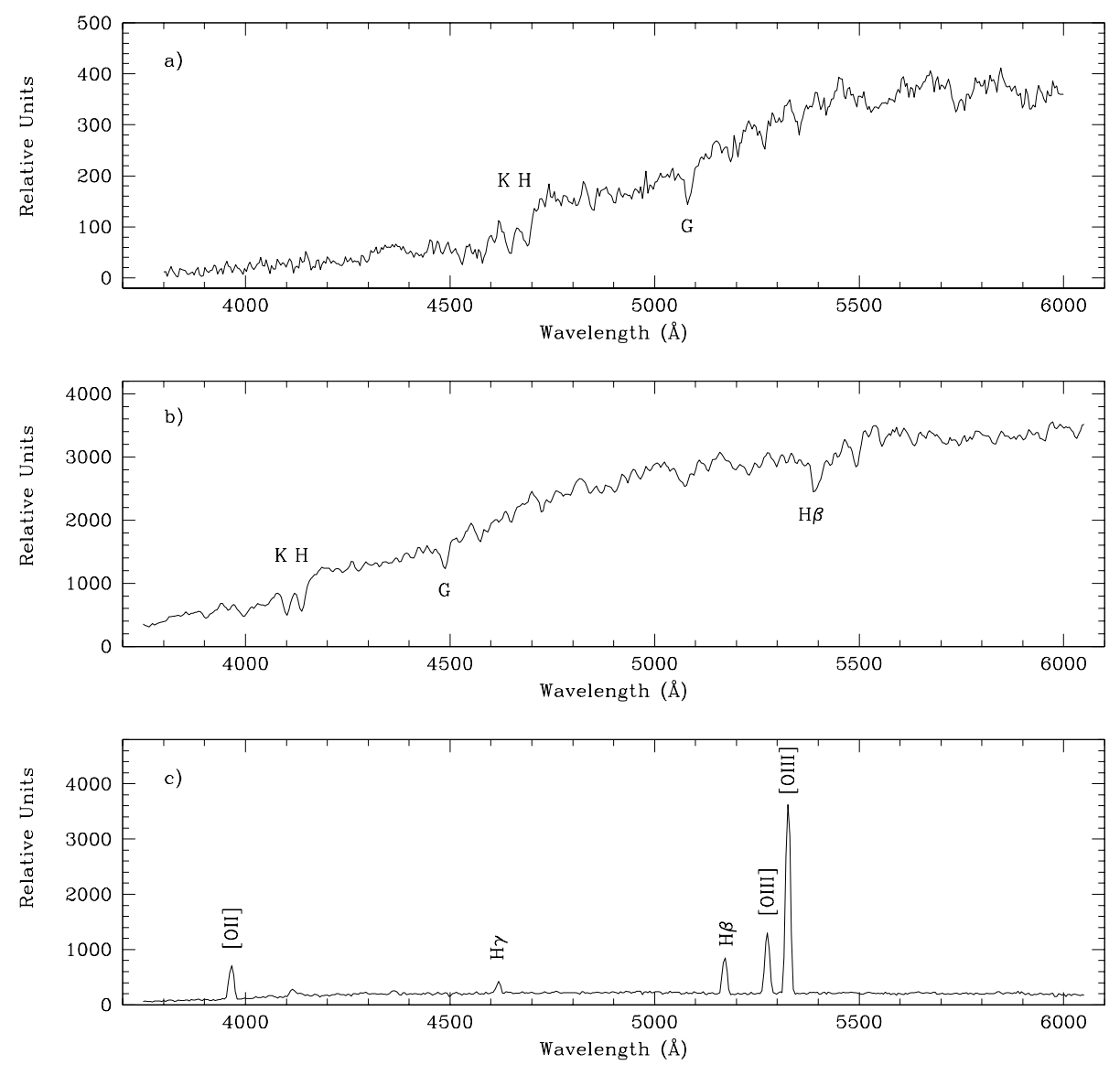

Fig. 1. Three examples of spectra: a) Standard quality spectrum $(R=3.8)$ of a galaxy with $v_{\text {abs }}=54315 \pm 46 \mathrm{~km} / \mathrm{s}$. b) High quality spectrum $(R=20.2)$ of a galaxy with $v_{\text {abs }}=12764 \pm 20 \mathrm{~km} / \mathrm{s}$. c) Spectrum with prominent emission lines of a galaxy with $v_{\text {emiss }}=19110 \pm 10 \mathrm{~km} / \mathrm{s}$

there is a small overlap between the fields which results in the fact that some galaxies belong to two adjacent fields; the galaxies in the overlap areas have been assigned to the field whose centre is closer to the object position.

We observed a total of 4043 objects, corresponding to $\sim 90 \%$ of the parent photometric sample of 4487 objects. Out of the 4043 observed objects, 493 turned out to be stars and 207 have a too low signal-to-noise ratio to provide a reliable redshift (failed spectra). In the end, our final sample consists of a total of 3343 objects with reliable redshifts (3342 galaxies and 1 QSO).

The survey data are available in electronic form at the CDS via anonymous ftp to cdsarc.ustrasbg.fr (130.79.128.5) or via http://cdsweb.ustrasbg.fr/Abstract.html. In Table 3 we provide a sample page of the catalogue, which is sorted in right ascension. The columns contain the following information: Column (1): ESP galaxy number.

Column (2): OPTOPUS field number.

Column (3): Right Ascension (1950).
Column (4): Declination (1950).

Column (5): $b_{\mathrm{J}}$ magnitude.

Column (6): Heliocentric Radial Velocity from absorption lines in $\mathrm{km} / \mathrm{s}$.

Column (7): Associated Internal Error in $\mathrm{km} / \mathrm{s}$.

Column (8): Value of the $R$ parameter (Tonry \& Davis 1979) from cross correlation.

Column (9): Heliocentric Radial Velocity from emission lines in $\mathrm{km} / \mathrm{s}$.

Column (10): Associated Error in km/s.

The codes -9999 and -8888 in the velocity columns indicate stellar spectra (hence stars misclassified in the EDSGC) and spectra not useful for radial velocity measurements, respectively. Objects which have not been observed have a zero in the velocity columns. For two galaxies (\# 13489 = NGC 7410 and \# 17863) the reported velocities are from da Costa et al. (1991) and Metcalfe et al. (1989), respectively. The object \# 31954, with the velocity coded as 99999, is a quasar with $z \sim 1.174$. Finally, a few galaxies have a measure for $v_{\text {abs }}$ but have $R=0$ : 
Table 3. Sample page of the catalogue (the whole catalogue is available only in electronic form)

\begin{tabular}{|c|c|c|c|c|c|c|c|c|c|}
\hline \# object & \# field & $\begin{array}{r}\alpha \\
(1950)\end{array}$ & $\begin{array}{r}\delta \\
(1950)\end{array}$ & $b_{\mathrm{J}}$ & $\begin{array}{r}v_{\text {abs }} \\
(\mathrm{km} / \mathrm{s})\end{array}$ & $\begin{array}{r}\text { err } \\
(\mathrm{km} / \mathrm{s})\end{array}$ & $R$ & $\begin{array}{r}v_{\text {emiss }} \\
(\mathrm{km} / \mathrm{s})\end{array}$ & $\begin{array}{r}\text { err } \\
(\mathrm{km} / \mathrm{s})\end{array}$ \\
\hline 32986 & 147 & 003108.5 & -403842 & 19.32 & 56575 & 106 & 2.34 & 56509 & 60 \\
\hline 33018 & 147 & 003112.8 & -404328 & 19.15 & 0 & 0 & 0.00 & 37564 & 14 \\
\hline 34795 & 147 & 003116.4 & -403032 & 18.70 & 35523 & 47 & 7.42 & 0 & 0 \\
\hline 34801 & 147 & 003121.5 & -402311 & 18.37 & -9999 & 0 & 0.00 & -9999 & 0 \\
\hline 34800 & 47 & 003122.0 & -394642 & 18.76 & 32884 & 37 & 9.80 & 0 & 0 \\
\hline 34802 & 147 & 003130.3 & -403312 & 17.94 & 33643 & 30 & 11.30 & 0 & 0 \\
\hline 33049 & 147 & 003133.9 & -403505 & 19.19 & 33875 & 74 & 4.31 & 33775 & 71 \\
\hline 34805 & 47 & 003139.0 & -400312 & 18.39 & 28517 & 42 & 9.81 & 0 & 0 \\
\hline 33089 & 147 & 003145.7 & -401847 & 19.39 & 0 & 0 & 0.00 & 20619 & 20 \\
\hline 34810 & 147 & 003146.5 & $\begin{array}{lll}-40 & 17 & 19\end{array}$ & 17.98 & 19991 & 33 & 12.69 & 0 & 0 \\
\hline 34811 & 147 & 003148.3 & -401740 & 18.71 & 20177 & 20 & 11.66 & 0 & 0 \\
\hline 33091 & 147 & 003151.3 & -403153 & 19.25 & 34073 & 103 & 3.48 & 33945 & 41 \\
\hline 34820 & 47 & 003154.9 & -395046 & 18.72 & 33519 & 34 & 11.63 & 0 & 0 \\
\hline 33132 & 47 & 003209.9 & -400853 & 18.96 & 28486 & 54 & 4.18 & 28637 & 37 \\
\hline 33130 & 47 & 003212.0 & -400154 & 18.89 & 32838 & 49 & 6.50 & 32844 & 37 \\
\hline 34826 & 147 & 003215.0 & -403209 & 17.65 & 20032 & 64 & 5.51 & 0 & 0 \\
\hline 33163 & 47 & 003219.8 & -395956 & 18.98 & 0 & 0 & 0.00 & 26223 & 23 \\
\hline 34831 & 47 & 003220.3 & -395058 & 18.61 & -8888 & 0 & 0.00 & -8888 & 0 \\
\hline 33161 & 47 & 003221.4 & -395627 & 19.00 & 54961 & 117 & 1.70 & 0 & 0 \\
\hline 33168 & 147 & 003225.7 & -401932 & 18.92 & 20073 & 88 & 4.10 & 20115 & 42 \\
\hline 33164 & 47 & 003227.2 & -400158 & 19.34 & 73433 & 99 & 3.18 & 0 & 0 \\
\hline 33162 & 47 & 003227.3 & -395716 & 18.95 & 55121 & 56 & 6.91 & 0 & 0 \\
\hline 34832 & 47 & 003227.6 & -400617 & 18.63 & 33059 & 91 & 3.22 & 0 & 0 \\
\hline 33165 & 47 & 003228.3 & -400411 & 19.32 & 0 & 0 & 0.00 & 0 & 0 \\
\hline 33166 & 47 & 003228.9 & -400425 & 18.93 & 0 & 0 & 0.00 & 11749 & 14 \\
\hline 33169 & 147 & 003232.8 & -402206 & 18.81 & 0 & 0 & 0.00 & 20186 & 67 \\
\hline 33189 & 47 & 003233.4 & -395126 & 18.87 & -8888 & 0 & 0.00 & -8888 & 0 \\
\hline 34840 & 147 & 003234.1 & -402810 & 18.59 & 24768 & 59 & 5.45 & 0 & 0 \\
\hline 33220 & 48 & 003244.9 & -400327 & 19.32 & 73218 & 68 & 4.00 & 0 & 0 \\
\hline 34848 & 148 & 003247.0 & -402312 & 18.42 & -9999 & 0 & 0.00 & -9999 & 0 \\
\hline 33225 & 148 & 003253.3 & -403120 & 19.22 & 34012 & 98 & 4.20 & 0 & 0 \\
\hline 33223 & 148 & 003253.8 & -402504 & 18.97 & 61621 & 82 & 3.00 & 61736 & 55 \\
\hline 34849 & 148 & 003254.5 & -403005 & 18.65 & 34230 & 94 & 3.50 & 34191 & 37 \\
\hline 34850 & 148 & 003257.4 & -403254 & 18.41 & 0 & 0 & 0.00 & 0 & 0 \\
\hline
\end{tabular}

this fact indicates low quality spectra for which the crosscorrelation was "forced", choosing the correlation peak by hand.

In the case of multiple observations of the same galaxy, we report in Table 3 the best measurement only.

For what concerns the use of these data for scientific analyses, when a galaxy has both $v_{\text {abs }}$ and $v_{\text {emiss }}$ the choice of the velocity can be done on the basis of the minimum error: however, the differences between $v_{\text {abs }}$ and $v_{\text {emiss }}$ are so small that different choices do not produce appreciable effects on the results of most of the scientific analyses. Note that the velocity errors reported in Cols. (7) and (10) are formal errors: for the conversion factors from these internal errors to the true errors, see the discussion in Sect. 6.2.

\section{Analysis}

\subsection{Possible biases in the sample}

Statistical analyses of this sample have to take into account and, if necessary, to correct for possible biases induced by the fact that some stars are misclassified as galaxies in the photometric catalogue, not all spectra have produced a measurable redshift and not all objects have been observed.

We have verified that the magnitude distribution of the stars is consistent with that of the total sample and therefore does not bias any analyses.

The small fraction $(\sim 5 \%)$ of objects with unmeasurable redshift has various origins: $\sim 13 \%$ of them correspond to undeblended pairs (see Sect. 2) for which the object coordinates fall in between the two components, while most cases are due to not well connected fibers or 


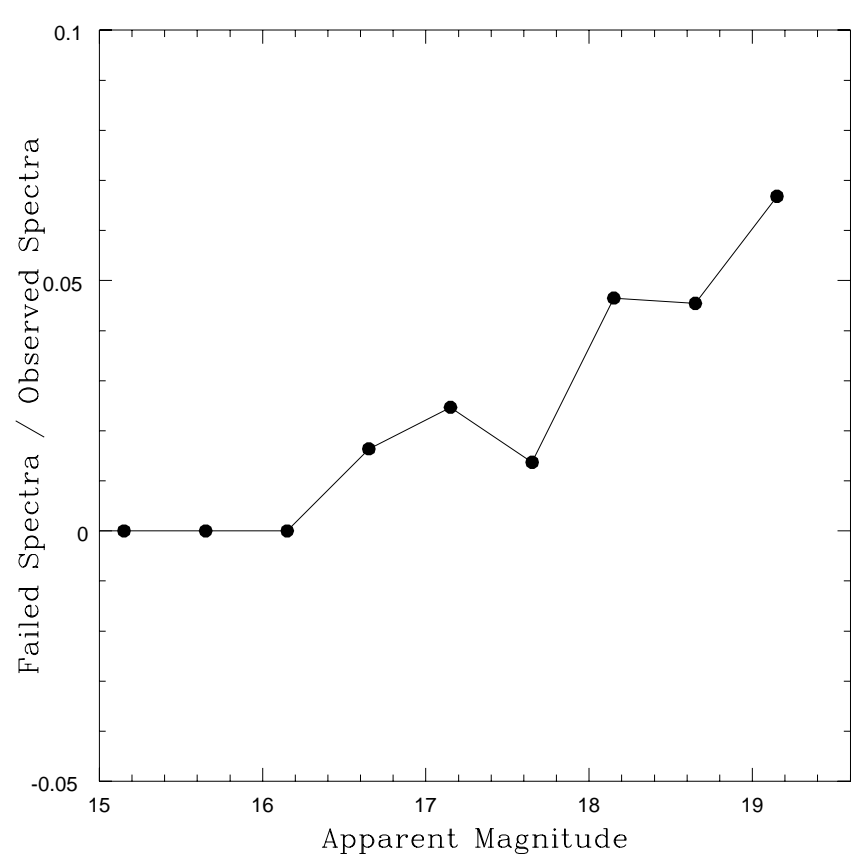

Fig. 2. Fraction of failed spectra over the total number of observed objects as a function of magnitude. Note that, even in the faintest bin, this fraction is always lower than $7 \%$

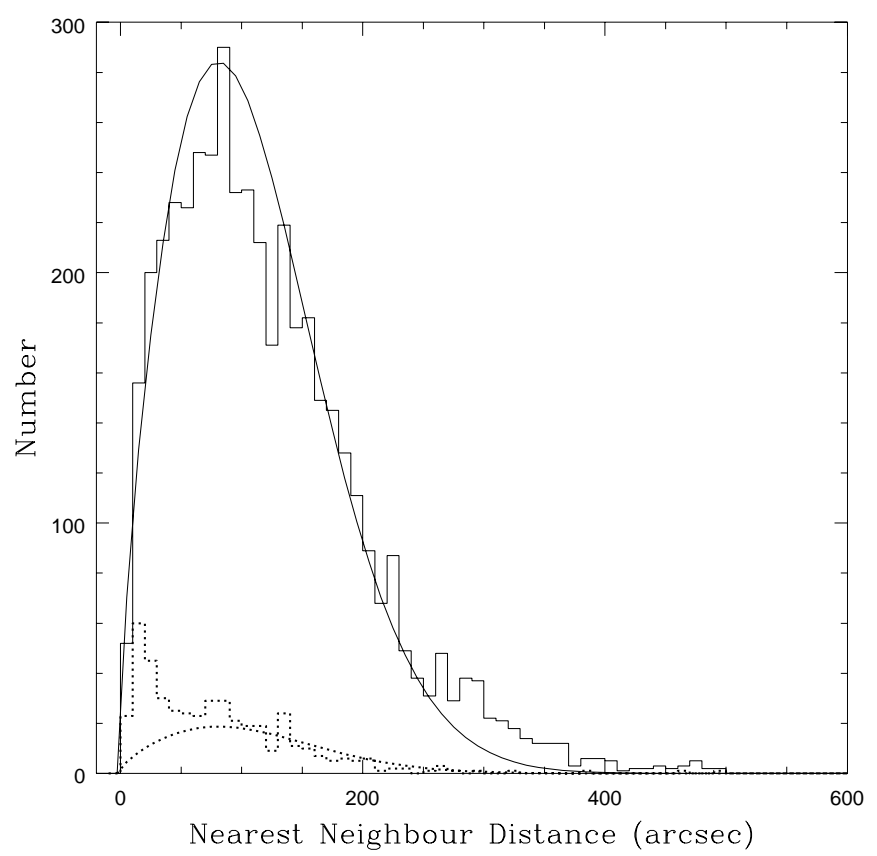

Fig. 3. Observed distribution of nearest neighbour distances $\left(d_{\mathrm{nn}}\right)$ for the entire photometric catalogue (4487 objects; solid histogram) and for the 444 objects which have not been observed spectroscopically (dashed histogram). The curves superimposed on the two histograms show the expected distributions of $d_{\mathrm{nn}}$ on the basis of the measured angular correlation function to observations with bad weather conditions. This kind of incompleteness is higher for fainter objects (see Fig. 2): note, however, that the maximum fraction of galaxies for which the spectra did not provide a useful $z$ determination is $\sim 7 \%$ for the faintest galaxies of our survey $\left(b_{\mathrm{J}}=19.4\right)$.

We have verified that the magnitude distribution of not-observed objects $(\sim 10 \%)$ is consistent with being a random extraction from the total sample. The main bias for such objects is introduced by the impossibility of observing, in a given OPTOPUS exposure, two objects closer than $\sim 25$ arcsec. Although the MEFOS observations and the repeated observations of the same fields have reduced somewhat this bias, it is still significantly present in the data. This is clearly seen in Fig. 3 which shows the observed distribution of nearest neighbour distances $\left(d_{\mathrm{nn}}\right)$ for the entire photometric catalogue (4487 objects; solid histogram) and for the 444 objects which have not been observed spectroscopically (dashed histogram). The curves superimposed on the two histograms show the expected distributions of $d_{\mathrm{nn}}$ on the basis of the measured angular correlation function. The excess of not-observed objects which have a neighbour at a distance smaller than 50 arcsec is $132 \pm 14$ (183 objects in the data while 54 would be expected); some, less significant excess (35 \pm 11 ) is present also for $d_{\mathrm{nn}}$ in the range 50-100 arcsec. Another way of characterizing the same bias is through the ratio between the not-observed objects and the total number of objects in the photometric catalogue as a function of $d_{\mathrm{nn}}$. This ratio is $0.31,0.130 .10,0.06$ for $d_{\mathrm{nn}}<30$, $30 \leq d_{\mathrm{nn}}<50,50 \leq d_{\mathrm{nn}}<100,100 \leq d_{\mathrm{nn}}$, respectively. Because of this bias, the spectroscopic catalogue can not be used in a straightforward way for studying, for example, the statistics of the number of close pairs or for analyzing the three dimensional correlation function on very small scales.

\subsection{Statistical analysis of velocity errors}

The errors on the velocities listed in Cols. (7) and (10) of Table 3 are the formal errors given by the IRAF tasks XCSAO and EMSAO for $v_{\text {abs }}$ and $v_{\text {emiss }}$, respectively. Their distributions are shown in Fig. 4: the median error is 64 $\mathrm{km} / \mathrm{s}$ for $v_{\text {abs }}$ and $31 \mathrm{~km} / \mathrm{s}$ for $v_{\text {emiss }}$. In order to have a better estimate of the true errors, we have analyzed the differences in the measured velocities $(\Delta v)$ of the galaxies which have been observed more than once (156 galaxies with two measurements of $v_{\text {abs }}$ and 64 galaxies with two measurements of $\left.v_{\text {emiss }}\right)$. For both these samples the histograms of $\Delta v$ normalized to the formal errors are significantly larger than expected if these formal errors were correct estimates of the true errors. By fitting these distributions with Gaussian curves, the dispersions of the best fitting Gaussians are 1.53 and 2.10 for $v_{\text {abs }}$ and $v_{\text {emiss }}$, respectively. This implies that, assuming that the ratio between the true and the formal errors is a constant for all 


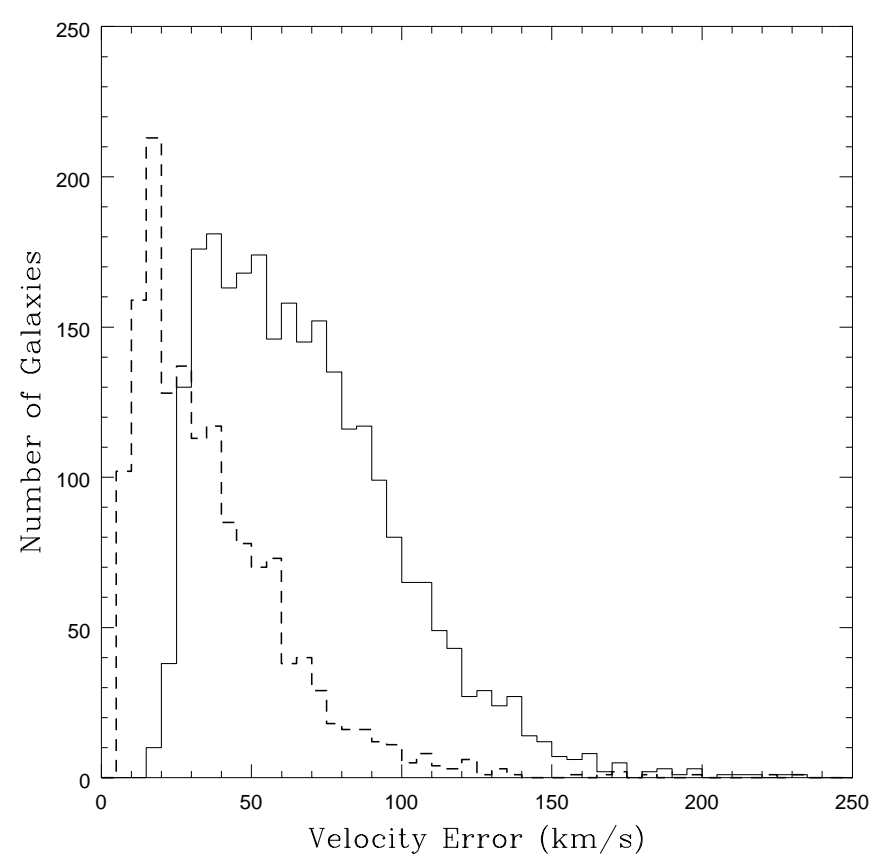

Fig. 4. Distribution of the errors on the measured velocity, for both $v_{\text {abs }}$ (solid histogram) and $v_{\text {emiss }}$ (dashed histogram)

measurements, the true errors can be estimated by multiplying the errors given in Table 3 by these factors.

The factor found for $v_{\text {abs }}$ is in agreement with other values reported in the literature from similar analyses: for instance, Bardelli et al. (1994) found a factor 1.87 from a comparison of two sets of OPTOPUS observations (45 galaxies) reduced with different packages (IRAF and MIDAS) and by different authors; Malumuth et al. (1992), using multiple observations of 42 galaxies reduced in the same way, found a factor 1.6.

\subsection{Systematic difference between $v_{\mathrm{emiss}}$ and $v_{\mathrm{abs}}$}

For about 750 galaxies we could measure the redshift both from absorption and from emission lines. The distribution of the difference $\left(v_{\text {abs }}-v_{\text {emiss }}\right)$ is well fitted by a Gaussian peaked at $\sim 100 \mathrm{~km} / \mathrm{s}$. We have performed a number of tests, and we can exclude a zero-point error. Also in the Las Campanas redshift survey a similar systematic difference between the absorption and the emission velocities has been found, and the authors have chosen to correct for it by using a separate average template with large Balmer lines to fit emission line galaxies (Shectman et al. 1996). They suggest that the systematic effect is mainly due to the blend between $\mathrm{H} \epsilon$ and $\mathrm{CaH}$ lines. On the other hand, we have decided to use in any case the best-fitting template, for sake of homogeneity in the redshift measures. A more general discussion about this point will be found in Cappi et al. (1998).

\section{Summary}

We have described in detail the data of the ESP galaxy redshift survey, which extends over about 23 square degrees, in a region near the South Galactic Pole. The survey is $\sim 85 \%$ complete to the limiting magnitude $b_{\mathrm{J}}=19.4$ and consists of 3342 galaxies with redshift determination. Although not all galaxies have been observed and not all spectra have produced a measurable redshift, we have shown that these facts do not introduce any bias in the final spectroscopic sample. The only significant bias still remaining in the sample is due to the fact that close pairs of galaxies could not be observed in a single OPTOPUS (or MEFOS) observation. For this reason the fraction of not-observed objects is significantly higher than average for objects which have a companion in the photometric catalogue at a distance smaller than about 50 arcsec.

For all galaxies we have determined, when possible, both absorption and emission velocities. The median formal errors on the velocities are 64 and $31 \mathrm{~km} / \mathrm{s}$ for the absorption and emission velocities, respectively. Analysis of the velocity measurements of the galaxies which have been observed more than once shows, however, that these formal errors are significant underestimates of the "true" errors. In first approximation the true errors can be obtained by multiplying the formal ones by factors of the order of 1.5 and 2.1 for $v_{\text {abs }}$ and $v_{\text {emiss }}$, respectively.

The data of the catalogue, available in electronic form at the the CDS via anonymous ftp to cdsarc.ustrasbg.fr (130.79.128.5) or via http://cdsweb.ustrasbg.fr/Abstract.html, provide all the information which is needed (i.e. positions, magnitudes, velocities, completeness) for statistical analyses of this sample, as for example the estimate of the luminosity function and mean galaxy density (Zucca et al. 1997).

Acknowledgements. This work has been partially
supported through NATO Grant CRG 920150 ,
EEC Contract ERB-CHRX-CT92-0033, CNR
Contract 95.01099.CT02 and by Institut National
des Sciences de l'Univers and Cosmology GDR.
It is a pleasure to thank the support we had from the
ESO staff both in La Silla and in Garching. In particular,
we are grateful to Gerardo Avila for his advice and his help
in solving every instrumental problem we have been facing
during this project. We also thank M. Kurtz and D. Mink who
kindly provided the RVSAO package before the official release
in IRAF.

\section{References}

Avila G., D'Odorico S., Tarenghi M., Guzzo L., 1989, The Messenger 55, 62

Avila G., et al., 1995, SPIE 2476, 77

Bardelli S., et al., 1994, MNRAS 267, 665

Cappi A., et al., 1998, A\&A (in press) (Paper IV)

Cuby J.-G., Mignoli M., 1994, SPIE 2198, 98 
da Costa L.N., et al., 1991, ApJS 75, 935

Felenbok, P., Guerin, J., Fernandez, A., Cayatte, V. Balkowski, C., Kraan-Korteweg, R.C., 1997, Exper. Astron. 7, 65

Heydon-Dumbleton N.H., Collins C.A., MacGillivray H.T., 1988, in Large-Scale Structures in the Universe, Seitter W., Duerbeck H.W. and Tacke M. (eds.). Springer-Verlag, p. 71

Heydon-Dumbleton N.H., Collins C.A., MacGillivray H.T., 1989, MNRAS 238, 379

Horne K., 1986, PASP 98, 609

Kurtz M.J., Mink D.J., Wyatt W.F., Fabricant D.G., Torres G., Kriss G., Tonry J.L., 1992, in Astronomical Data Analysis Software and Systems I, ASP Conf. Ser. 25, Worral D.M., Biemesderfer C. \& Barnes J. (eds.) p. 432

Lund G., 1986, OPTOPUS - ESO Operating Manual No. 6,
ESO, Garching bei München

MacGillivray H.T., Stobie R., 1984, Vistas Astron. 27, 433

Malumuth E.M., Kriss G.A., Dixon W.V.D., Ferguson H.C., Ritchie, C., 1992, AJ 104, 495

Metcalfe N., Fong R., Shanks T., Kilkenny D., 1989, MNRAS 236, 207

Mink D.J., Wyatt W.F., 1995, in Astronomical Data Analysis Software and Systems IV, ASP Conf. Ser. 77, Shaw R.A., Payne H.E. \& Hayes J.J.E. (eds.) p. 496

Parry I.R., Carrasco E., 1990, SPIE 1235, 702

Shectman S.A., Landy S.D., Oemler A., Tucker D.L., Lin H., Kirshner R.P., Schecter P.L., 1996, ApJ 470, 172

Tonry J., Davis M., 1979, AJ 84, 1511

Vettolani G., et al., 1997, A\&A 325, 954 (Paper I)

Wyse R.F.G., Gilmore G., 1992, MNRAS 257, 1

Zucca E., et al., 1997, A\&A 326, 477 (Paper II) 